\title{
Quelques Clinocerini (Diptera, Empididae, Hemerodromiinae) nouveaux ou mal connus des Pyrénées
}

\author{
F. Vaillant: \\ G. Vinçon ${ }^{2}$
}

ITots clés : Diptera, Empididae, nouvelles espèces, larves aquatiques, Pyrénées.

Dans les Hautes-Pyrénées, 29 especes de Clinocerini sont signalées, dont 24 ont été décrites antérieurement. L'une des 29 espèces, ainsi que 4 autres espèces nouvelles des Pyrénées-Atlantiques, sont décrites ici. Les caractères d'une espèce mal connue sont précisés.

The Clinocerini (Diptera, Empididae, Hemerodromilnae) of the Pyrenees

Keywords : Diptera, Empididae, new species, aquatic larvae, Pyrenees.

A description of 5 new species of Clinocerini from the Pyrenees with a redescription of one previously poorly characterized species are given.

Les Clinocerini sont de petits Diptères brunâtres à caractères assez uniformes, qui fréquentent les abords de sources ou de cours d'eau rapides. Ils sont tous entomophages. Les uns (Wiedemannia) se tiennent sur les pierres des torrents, mouillées par les embruns de cascades ou par le clapotis de l'eau ; les autres (Clinocera, Bergenstammia) sont posés sur des parois rocheuses suintantes. Les larves des Wiedemannia circulent sur et sous les pierres immer. gées des torrents et dans le sédiment grossier du fond ou des rives, de sorte qu'on en recueille fréquemment avec le filet Surber. Les larves des Cli. nocera et des Bergenstammia font partie de biocénoses madicoles. Les larves des Clinocerini sont également entomophages.

On a décrit près de 200 espèces de Clinocerini de l'hémisphère boréal, mais seules celles d'Europe centrale sont bien connues. Dans la récente révision des espèces animales aquatiques d'Europe,

1. Laboratoire de Zoologie. Université scientifique et médicale de Grenoble, 38402 Saint-Martin-d'Hères Cédex ; France.

2. Laboraloire d'Hydrobiologit, UA CNRS 695, Université Paul Sabatier, 1 18, route de Narbonne 31062 Toulouse Cédex ; France.
29 espèces de Clinocerini sont signalées des Pyrénées (Vaillant 1978), mais 5 d'entre elles sont des nomina nuda; l'une de ces dernières sera décrite ici, ainsi que 4 autres, qui sont toutes nouvelles.

La classification des Clinocerini a été basée jusqu'ici essentiellement sur les caractères de la chètosité du corps et des pattes, mais ceux-ci se montrent très insuffisants, de sorte que nous ferons porter l'accent sur les caractères de la nervation alaire et sur ceux des pièces génitales mâles.

Il est peu pratique de représenter une aile pour chaque espèce décrite; en effet, si l'image n'est pas assez grande, les caractères importants n'apparaissent pas ; si elle l'est, Ja représentation de l'aile se fait au détriment de celle des pièces génitales mâles, pour lesquelles plusieurs figures détaillées doivent obligatoirement être données. Il nous a paru préférable de remplacer le dessin de l'aile par une série de chiffres ou "indices", dont les uns sont des rapports de longueur et dont les autres font apparaître les positions respectives de certains points. Pour obtenir ces chiffres, l'observateur fera, sur une grande feuille de papier, à l'aide d'une chambre claire et au crayon, une représentation de toutes les 

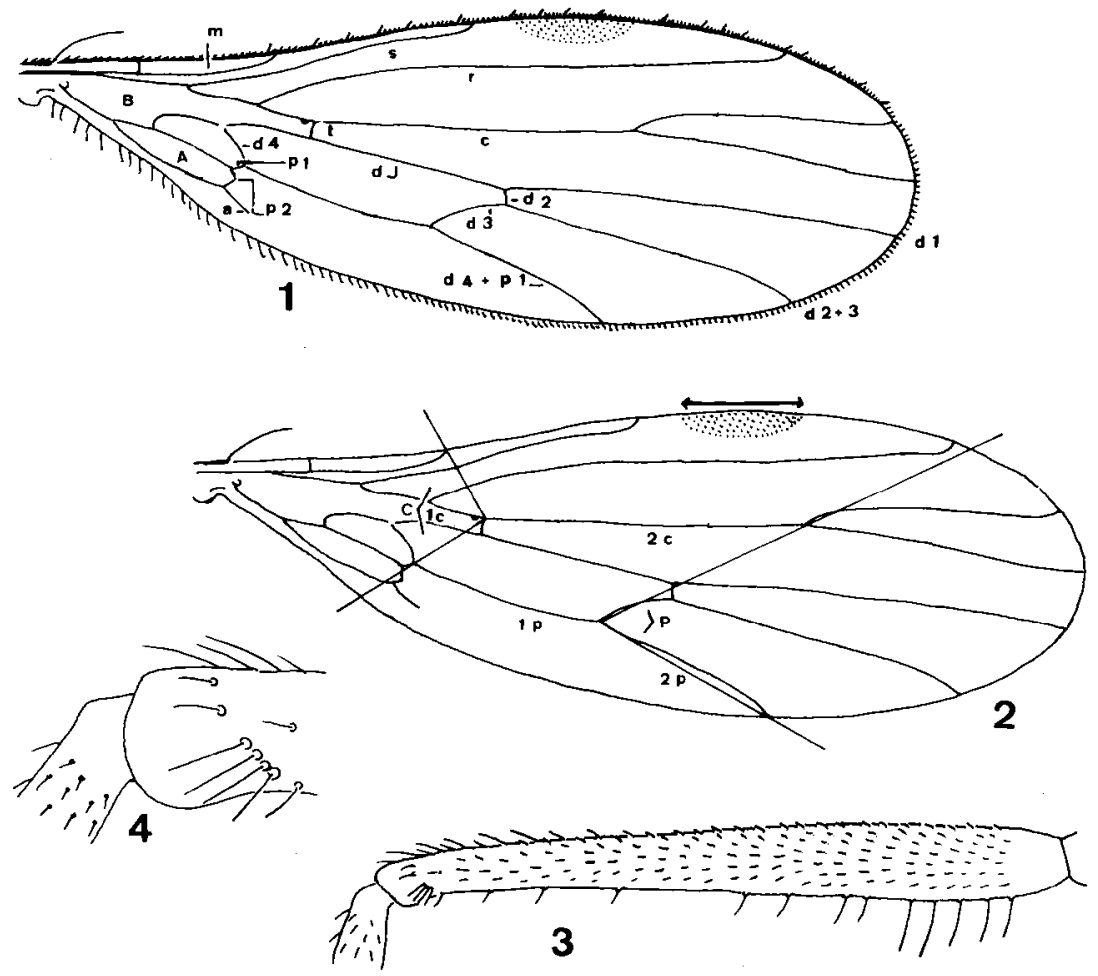

Fig. 1-4 : imagos mâles - 1 et 2 : Clinocera subplectrum n. sp., aile - 3 et 4 : Clinocera zwicki n. sp. - 3 : Fémur I droit, face antérieure - $4:$ détail de la partie distale du même fémur.

a : nervure anale $-c$ : nervure cubitale $-d$ : nervure discale $-m:$ nervure médiastinale $-p:$ nervure posticale $-r:$ nervure radiale - $s$ : nervure subcostale - $t:$ nervure transverse discale - A : cellule anale - $\mathrm{B}$ : première cellu le basale - $\mathrm{C}$ : angle cubital $\cdot \mathrm{P}$ : angle postical $-1 \mathrm{c}$ : premier segment de la nervure cubitale $-2 \mathrm{c}$ : deuxieme segment de la nervure cubitale - $1 p$ : premier segment de $d_{4}+p_{1}-2 p$ : deuxième segment de $d_{4}+p_{1}$. 
nervures de l'aile (fig. 1). Plusieurs segments de nervures seront alors mesurés sur le dessin, en joignant par une droite les deux extrémités de chaque segment. Ensuite certains points seront repérés avec précision sur la figure de l'ajle, là où se rejoignent 2 ou 3 nervures; certains de ces points setont réunis par une droite et les angles formés par deux droites déterminées seront mesurés avec un rapporteur. Pour un observateur ayant quelque habitude, le dessin de l'aile et l'ensemble des opérations que nous venons d'indiquer ne demandent pas 10 minutes. I1 est tout à fait déconseillé de faire des mesures directes avec un micromètre oculaire ou avec un micromèt re objectif, car on augmente alors considérablement les risques d'erreurs, sans gagner du temps. Voici les rapports et les angles caractéristiques:

1) rapport stigmatique : rapport ent re la longueur de l'aile, comptée à partir du grand chète basal porté par la nervure costale, et la longueur de la tache stig. matique, lorsqu'il y en a une (fig. 2).

2) rapport cubital: rapport entre la longueur du deuxième segment $2 \mathrm{c}$ de la nervure cubitale et celle du premier segment $1 \mathrm{c}$ de cette même nervure.

3) rapport postical: rapport entre la longueur $\mathrm{du}$ deuxième segment de $d_{4}+p_{1}$, que nous désignerons par $2 \mathrm{p}$, et celle du premier segment de $\mathrm{d}_{4}+\mathrm{p}_{1}$, que nous désignerons par $1 \mathrm{p}$.

4) angle cubital : angle $\mathrm{C}$, compté du côté de la racine de l'aile, formé d'une part par la droite joignant l'extrémité du premier segment de la nervure cubitale et l'extrémité de la nervure médiastinale, d'autre part par la droite joignant l'extrémité du premier segment de la nervure cubitale et le point de confluence de $\mathrm{d}_{4}$ et de $\mathrm{p}_{1}$.

5) angle postical : angle $P$, compté du côté de l'apex de l'aile, formé d'une part par la droite joignant la fourche $d_{3}-d_{4}+p_{1}$ et la fourche cubitale, d'autre part par la droite joignant la fourche $d_{3}-d_{4}+$ $\mathrm{p}_{1}$ à l'extrémité de la nervure $\mathrm{d}_{4}+\mathrm{p}_{1}$.

Il y a lieu de préciser que la nomenclature de l'aile est empruntée à J.-E. Collin, sans la moindre modification.

Rapport cubital, rapport postical, angle cubital et angle postical varient entre des limites étroites chez une même espèce et par contre sont souvent très différents pour des espèces distinctes, qui ont par ailleurs une chètosité et des pièces génitales fort voisines; ceci justifie donc tout à fait l'emploi de tels
" indices $"$. L'angle cubital se montre particulièrement caractéristique, car il traduit l'importance de la première cellule basale $\mathbf{B}$ par rapport à l'ensemble de l'aile.

Le lecteur pourra remarquer qu'aucun compte n'est tenu de la nervure discale pour l'un ou pour l'autre des "indices". En effet la nervure transverse $d_{2}$ peut avoir une longueur et une position très diverses chez une même espèce ; elle peut faire défaut, ou bien il peut $y$ avoir deux transverses $d_{2}$ délimitant une petite cellule rectangulaire. Chez certaines espèces de Wiedemannia, d et $\mathrm{d}_{3}$ se réunissent pour former un segment de nervure longitudinale de longueur variable, puis se séparent à nouveau pour former les nervures $d_{1}$ et $d_{2}+3$.

Passons maintenant aux caractères des pièces génitales mâles ; la nomenclature est ici empruntée à $\mathrm{H}$. Ulrich, mais, du fait que cet auteur n'a étudié qu'une seule espèce de Clinocerini, nous avons dù, pour certaines de celles que nous avons décrites, proposer des interprétations, lesquelles ne sont pas étayées par une étude de la musculature.

Pour mieux comprendre l'architecture de l'armature génitale mâle chez les Clinocerini, nous prendrons pour exemple Wiedemannia wachtli (Mik), espèce pour laquelle cette architecture est proche de celle indiquée par Ulrich pour Wiedemannia ouedorum Vaillant (fig. 9.12 et 16). La "partie fixe" de l'armature génitale est constituée de deux pièces grossièrement semi-sphériques, fortement chitinisées sur leur face externe et à téguments minces sur leur face interne ; Ulrich estime que chacune de ces pièces représente un gonocoxite $(\mathrm{gc})$ intimement soudé à une partie de l'épandrium ; ces deux pièces sont réunies l'une à l'autre du côté antérieur par une courte et épaisse bande chit ineuse, que nous appellerons " pont tergal "(pt). Chaque gonocoxite s'articule latéralement avec l'hypandrium (hy), pièce fortement chitinisée en forme de dóme légèrement courbé et se prolongeant par la partie libre de l'aedeagus (ae). Sur chaque gonocoxite s'attache en arrière une petite pièce foliacée et cilièe, qui est ici mobile par rapport au gonocoxite, alors qu'elle ne l'est pas chez d'autres especes de Wiedemannia; Ulrich considère cette pièce comme un prolongement postérieur du gonocoxite (pg) (fig. 16). La partie libre de l'aedeagus est maintenue en place entre les deux gonocoxites par les prolongements postérieurs de ceux-ci et coulisse contre le sclérite du 


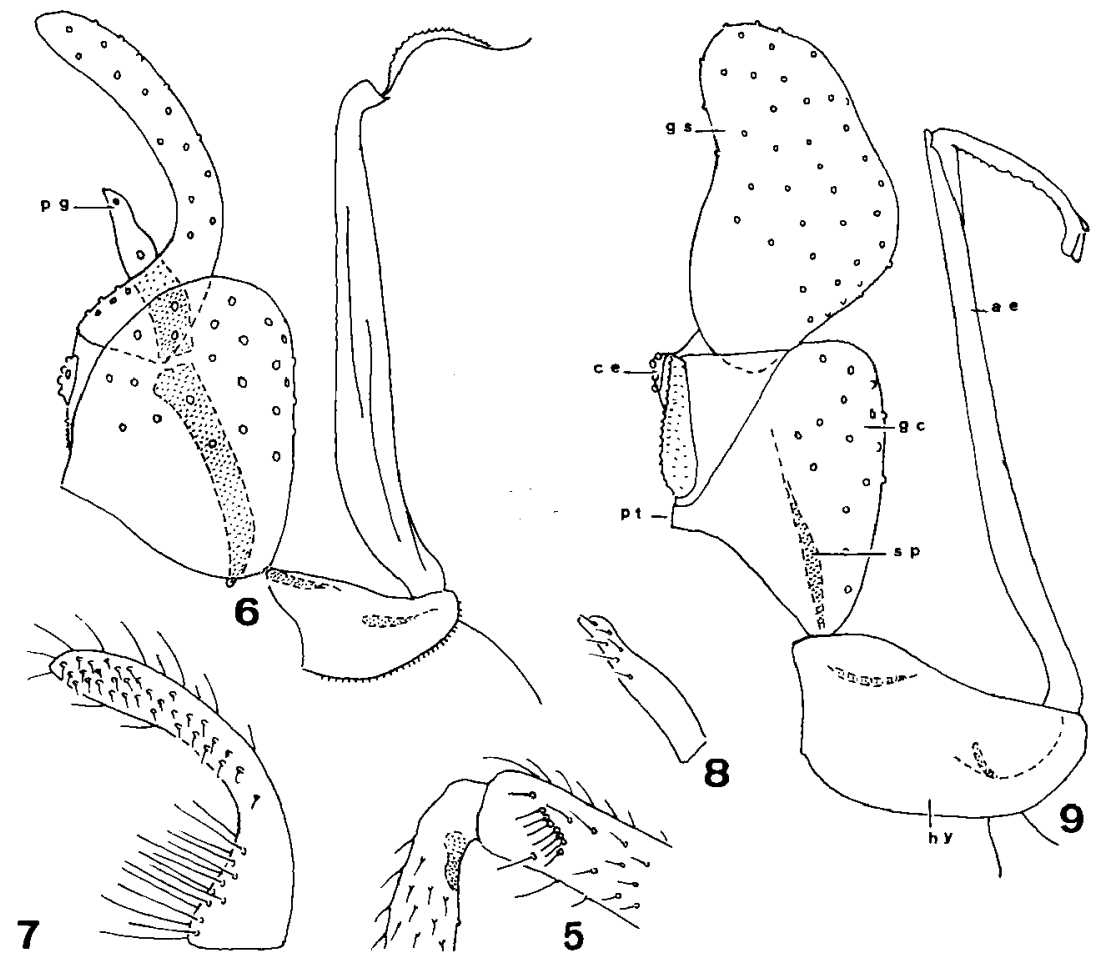

Fig. 5-9 : imagos mâles - 5 - 8 : Clinocera subplectrum n. sp. - 5 : partie distale du fémur I droit, face antérieure - $6:$ genitalia, profil - $7:$ gonostyle droit, face interne $-8:$ prolongement postérieur du gonocoxite droit, face interne - $9:$ Wiedemannia wachtli (Mik), genitalia, profil.

ae : aedeagus - ce : cerque - gc : gonocoxite - gs : gonostyle - hy : hypandrium - pg : prolongement postérieur du gonocoxite - pt : pont tergal - sp : sclérite du proctiger, vu par transparence. 
(5)
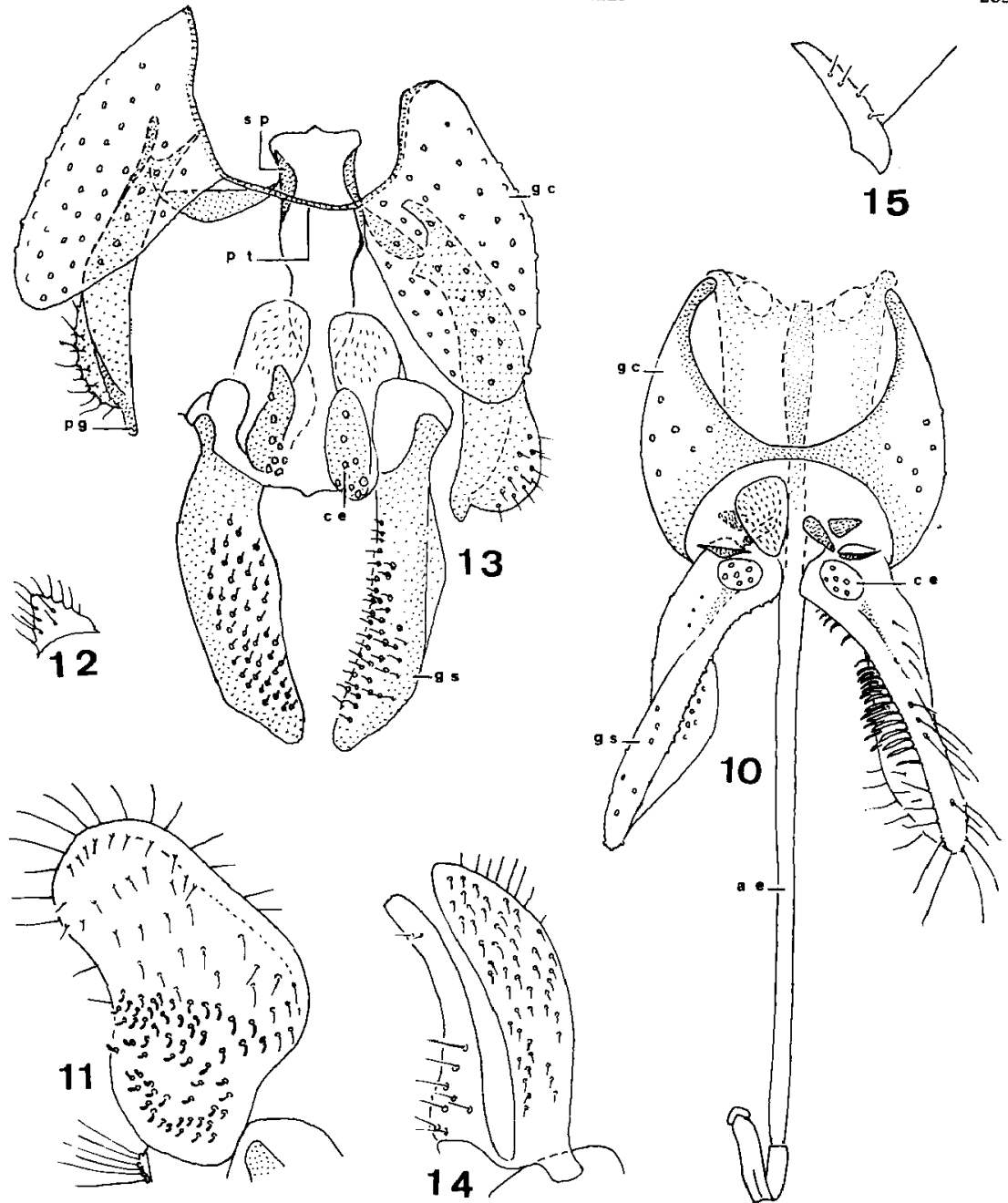

Fig. 10 - 15 : imagos mâles - 10 - 12 : Wiedemannia wachti (Mik), - 10 : genitalia, face dorsale, les gonocoxites rabattus contre l'hypándrium - 11 : gonostyle droit, face interne - 12 : prolongement postérieur du gonocoxite droit, face interne - 13 : Clinocera schremmeri (Vaillant), genitalia disséqués et étalés, en vue antérieure ; l'hypandrium n'a pas été figuré - 14 et 15 : Clinocera zwicki n. sp. - 14 : gonostyle droit, face inteme - 15 : prolongement postérieur du gonocoxite droit, face interne.

ae : aedeagus - ce : cerque - gc : gonocoxite - gs : gonostyle - pg : prolongement postérieur du gonocoxite - pt : pont tergal - sp : sclérite du proctiger. 

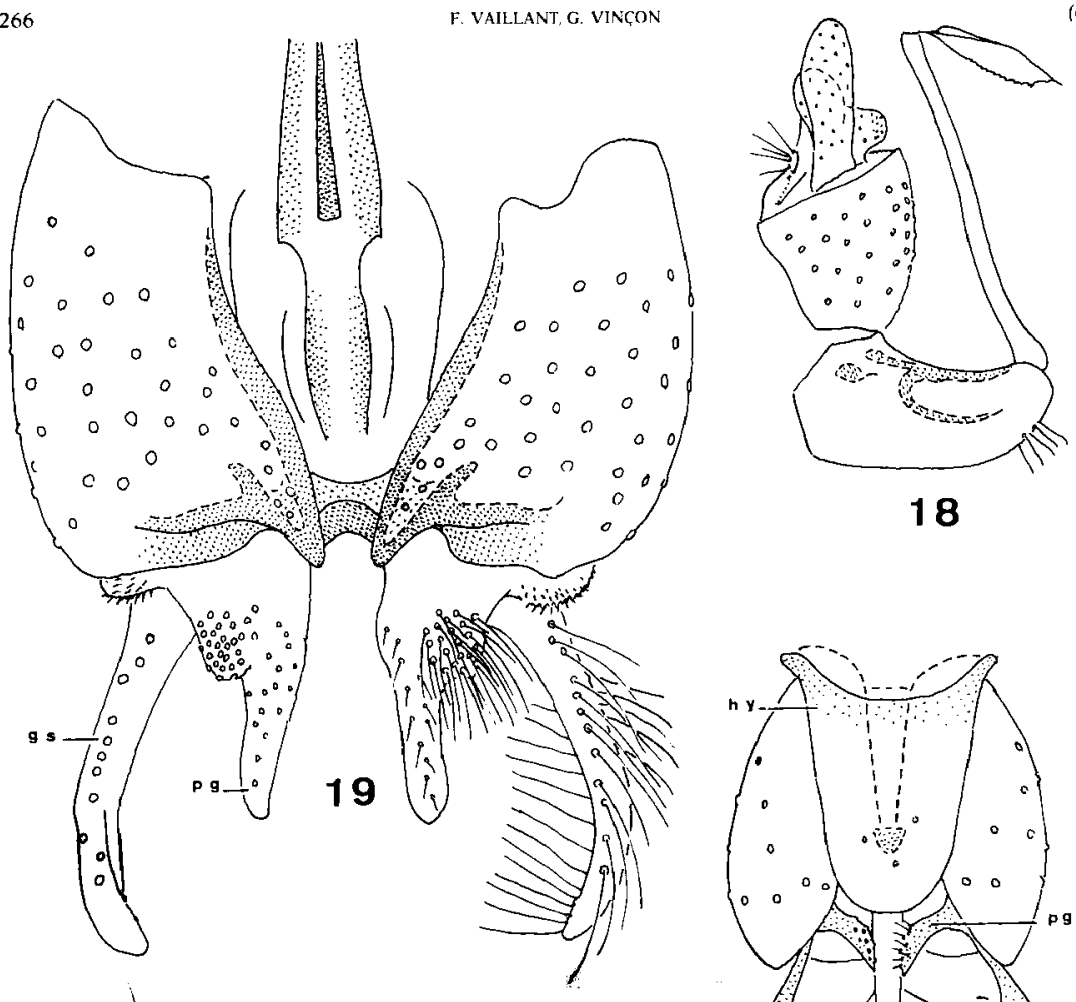

(6)
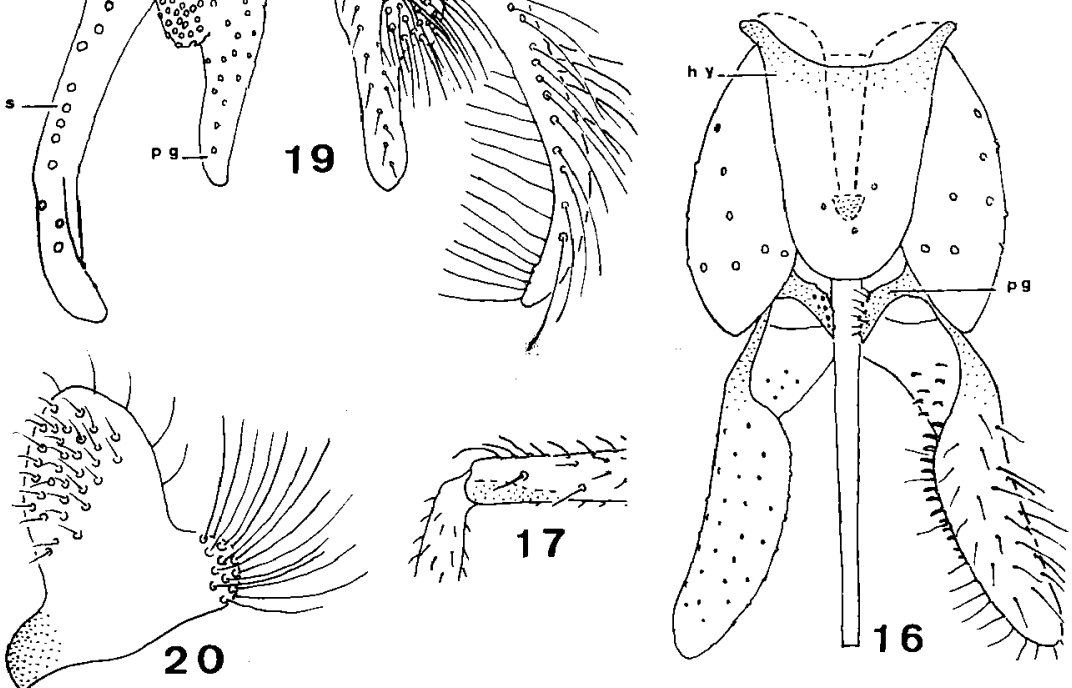

Fì. 16-20: imagos mâles - 16: Wiedemannia wachtli (Mik), genitalia, face ventrale, les gonocoxites rabattus contre l'hypandrium - 17-20:Bergenstammia pyrenaica n. sp. - 17: partie distale du fémur I gauche, face externe - $18:$ genitalia, profil - 19 : genitalia, face postërieure; l'hypandrium n'a pas été figuré - 20 : prolongement postérieur du gonocoxite droit, face interne. gs : gonostyle - pg : prolongement postérieur du gonocoxite - hy : hypandrium. 
proctiger (sp). Chaque gonocoxite se prolonge par un gonostyle (gs) lamelliforme et garni de courtes soies arquées, les rétinacles, sur sa face interne; les gonostyles sensu Ulrich étaient considérés comme les cerques par R. Bährmann et d'autres auteurs, qui ne se compromet taient pas, les désignaient sous le nom de lamelles terminales (E.-O. Engel) ou de lamelle latérales (J.-E. Collin); en tenant compte de la position de l'anus, Ulrich estime que les véritables cerques (ce) sont ici deux petits sclérites ovalaires, garnis de chètes et situés sur une aire membraneuse en avant des gonocoxites et près de la base des gonostyles ; deux autres pièces, moins fortement chitinisées, couvertes de cils et situées juste en des. sous des deux petits sclérites, représenteraient peutêtre, selon Ulrich, une partie des cerques.

L'interprétation d'Ulrich peut être transposée à toutes les espèces de Wiedemannia, mais des dou. tes apparaissent lorsqu'il s'agit des Clinocera et des Bergenstammia.

Voyons tout d'abord le cas des Clinocera. Les gonostyles sont parfois simples, parfois bilobés et ont une position externe. Les prolongements postérieurs des gonocoxites, assez petits chez certaines espèces (fig. 26), ont par contre un grand développement chez d'autres (fig. 13) et sont toujours mobiles par rapport aux gonocoxites; ils sont raccordés chacun au sclérite du proctiger par l'intermédiaire d'un apodème en forme de lame (fig. 13).

Le cas des Bergenstammia est plus embarrassant, car les prolongements postérieurs des gonocoxites, assez courts, font corps avec eux et sont fortement chitinisés : les cerques, ovalaires, sont représentés, mais chaque gonocoxite a deux expansions lamellaires de grande taille, qui s'articulent sur lui; la plus externe de ces expansions peut être considérée comme un gonostyle, d'autant plus qu'elle porte, chez certaines espèces, des rétinacles sur sa face interne. Mais que représente l'autre expansion, qui est généralement bilobée et qui est placée un peu en arrière du gonostyle? On peut admettre que le prolongement postérieur de chaque gonocoxite est décomposé ici en une partie fixe, fortement chitinisée, et en une partie mobile et foliacée, de plus grande taille. C'est cette dernière interprétation que nous retiendrons provisoirement.

La plupart des descriptions anciennes de Clinocerini n'étaient pas accompagnées de figures des genitalia mâles ou bien ces figures étaient très sommaires et représentaient la partie terminale du corps d'un spécimen sec, piqué sur épingle. Aussi est-il parfois difficile d'être sûr d'une détermination spécifique, lorsqu'on ne dispose pas du type ou d'un paratype et qu'on n'a pas la possibilité de faire un montage de ses pièces génitales entre lame et lamelle, pour mettre en évidence les details de l'ornementation de ces pièces.

Comme il est maintenant bien établi que ce sont les genitalia mâles qui fournissent les meilleurs caractères taxonomiques à l'intérieur d'un genre ou même d'une tribu d'Empididae, il est évident que des montages des genitalia, nettoyés dans un bain de potasse puis par un passage dans de l'acide acétique, doivent être effectués pour chaque spécimen que l'on désire déterminer. Un montage dans le baume du Canada est nettement à conseiller, car cette substance à l'avantage de ne pas trop diminuer de volume en séchant; par conséquent la lamelle n'écrase pas les pièces en les déformant; on peut même, si l'on désire garder une certaine épaisseur, introduire dans le baume sous la lamelle des fragments de verre. Lorsqu'on ne dispose que d'un seul spécimen, il faut l'utiliser pour obtenir une vue de profil des genitalia. Sur la mème lame on met alors trois lamelles; l'une recouvre les deux ailes, séparées du tronc, la seconde les genitalia et la troisième le reste du corps disposé de façon à être vu latéralement et à ce que les pat tes soient étalées. Les genitalia doivent ètre mis exactement de profil et la partie libre de l'aedeagus doit être dégagée de sa gouttière et tirée en arrière, pour que l'on puisse la voir sur toute sa longueur. Si l'on dispose d'un deuxième spécimen, il faut monter ses genitalia en vue dorsale, le plan de symétrie de l'ensemble étant rigoureusement perpendiculaire à la surface de la lame. En mettant la lamelle, on repousse les gonocoxites vers l'arrière et on les amène contre l'hypandrium (fig. 10 et 16) ou bien on détache l'hypandrium, que l'on place à côté du reste de l'appareil génital, lequel est seul à être représenté (fig. 19 et 22).

Dans les descriptions qui vont suivre, nous ne ferons généralement pas état des caractères de coloration du corps ; ceux-ci diffèrent en effet trop peu d'une espèce à une autre pour présenter un grand intèrêt.

Tous les Clinocerini dont il va être question maintenant ont été capturés en bordure d'un ou de plusieurs tributaires du gave d'Ossau dans les Pyrénées Atlantiques. 

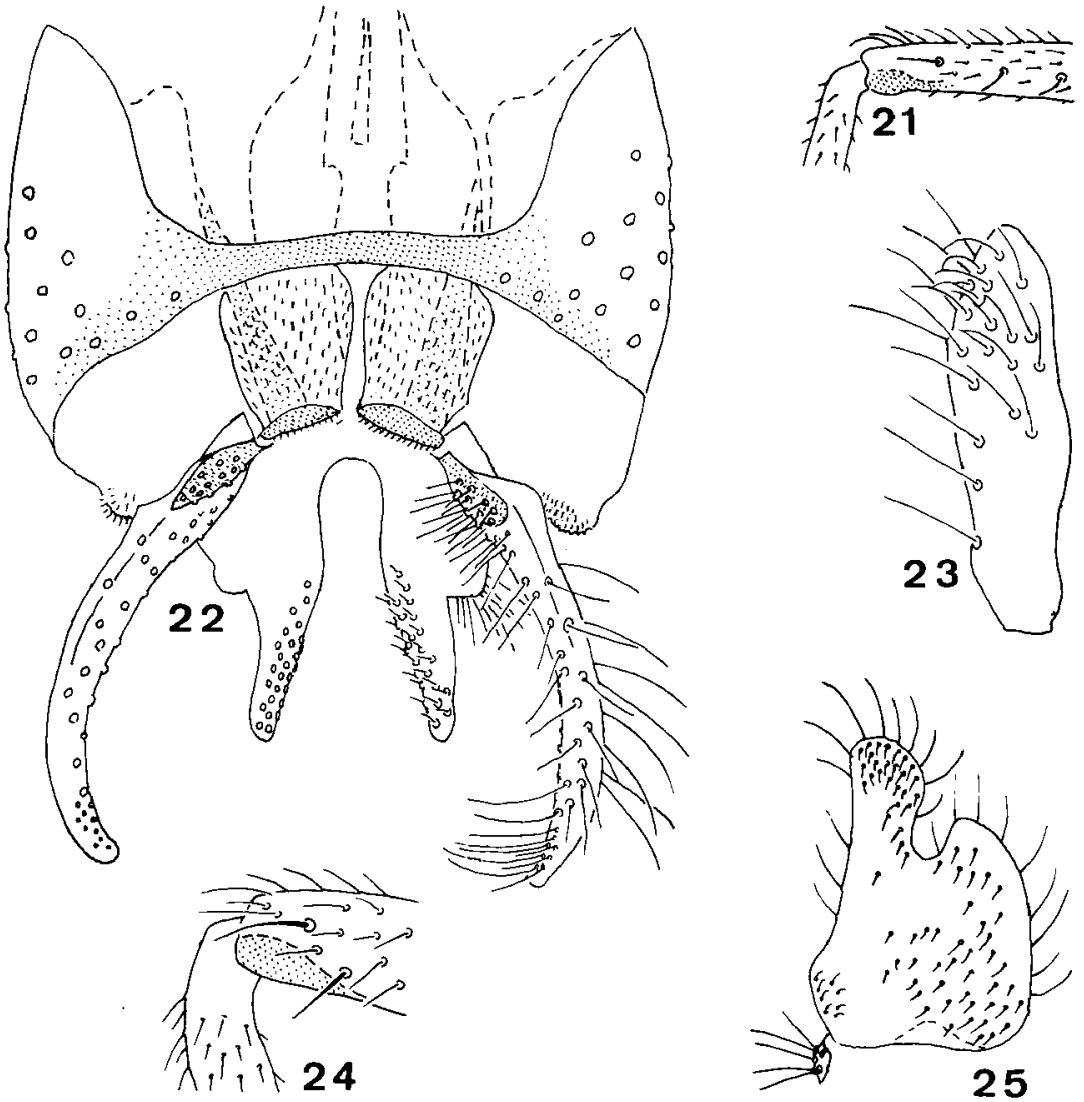

Fig. 21 - 25 : imagos mâles - $21-23:$ Bergenstammia pyrenaica n. sp. -21 : partie distale du fémur I droit, face antérieure - 22 : genitalia, face antérieure ; l'hypandrium n'a pas été figuré - 23 : gonostyle droit, face interne - 24 et $25:$ Wiedemannia berthelemyi n. sp. - 24 : partie distale du fémur I droit, face antérieure - 25 : gonostyle droit, face interne. 

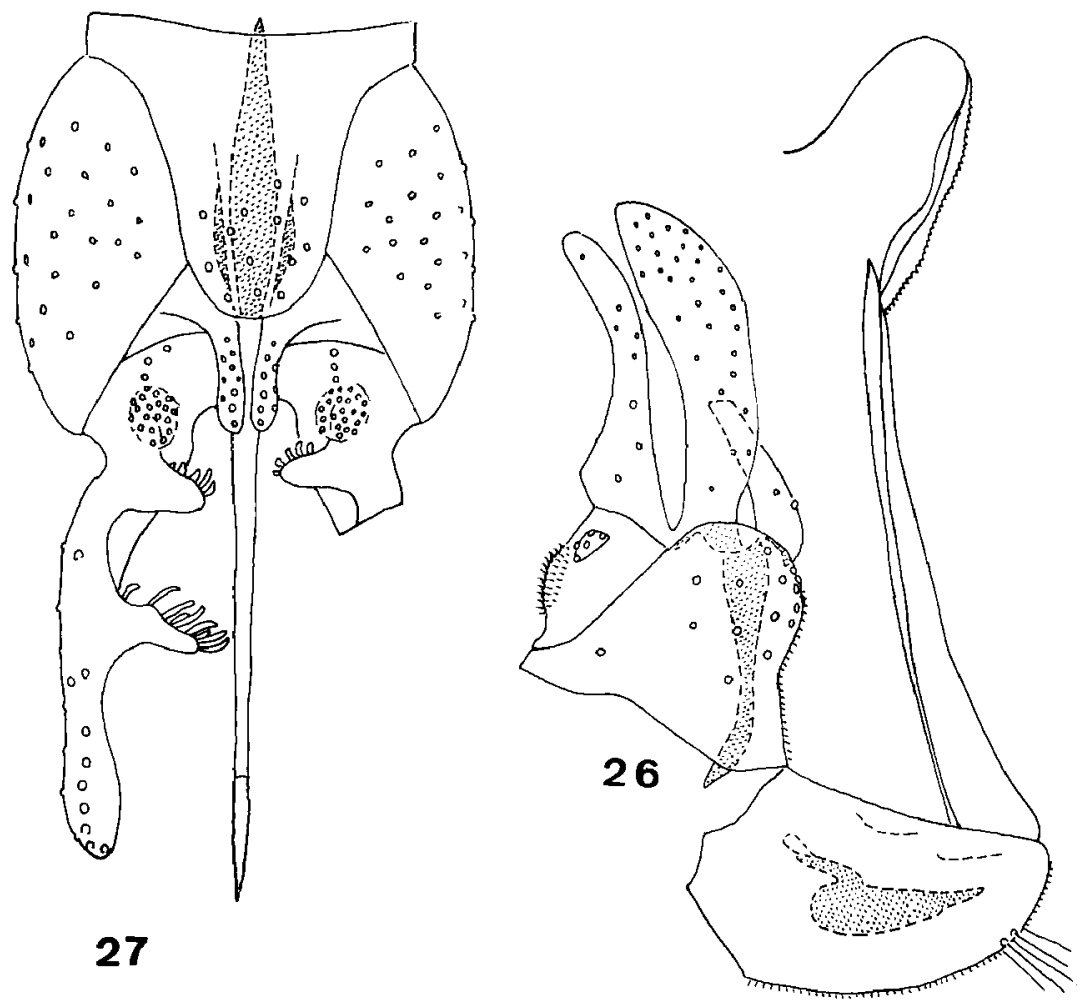

Fig. 26 et 27 : imagos mâles - 26: Clinocera zwicki n. sp., genitalia, profil - 27 : Wiedemannia digitata n. sp., genitalia, face ventrale, les gonocoxites rabattus contre l'hypandrium. 


\section{Clinocera (Phaeobalia) zwickl n. sp.}

Imago $\sigma$ : Coloration du corps peu différente de celle de Cl. (Ph.) lyneborgi Vaillant et Chvála. De chaque côté du mésonotum, 2 acrosticales tout à fait en avant, $4 \mathrm{dc}, 1$ chète huméral, 1 présutural, 2 chètes dans le triangle sutural, 1 supraalaire. Deux chètes scutellaires seulement. Fémur I ( $/$ ig. 3) : 9 ou 10 soies ventrales alignées sur la $1 / 2$ proximale, dont les plus grandes sont longues comme les $2 / 3$ du tra. vers du fémur, en outre 3 ou 4 chètes ventraux aljgnés sur la $1 / 2$ distale du fémur, 1 préapical ventral et 1 préapical ventro-postérieur : le peigne distal (fig. 4) comprend seulement 4 éléments et il y a un autre chète de même longueur en dessous du peigne. Aile: pas de tache stigmatique, ni de parties enfumées sur la membrane ; rapport cubital : 5,62 ; rapport postical : 0,95 ; angle cubital : $117^{\circ}$; angle postical : $52^{\circ}$; nervure $\mathrm{p}_{2}$ incurvée irrégulièrement ; deuxième segment de la nervure anale bien distinct et aussi long que la nervure $d_{1.4} ;$ un court rameau récurrent part de $c_{1} ;$ l'extrémité de $c_{2}$ est un peu plus proche de l'apex de l'aile que celle de $d_{1}$. Genitalia: gonostyles bifides et dont les deux branches sont de longueur à peu près égale (fig. 26) : la branche postérieure a des chètes internes assez. régulièrement répartis (fig. 14) ; prolongements postérieurs des gonocoxites petits et grèles (fig. 15): hypandrium court, avec 5 ou 6 chètes groupés (fig. 26).

Longueur de l'aile : 4,0 $4,1 \mathrm{~mm}$.

Un seul exemplaire, l'holotype, a été capturé le 22.9 .1985 près du ruisseau d'Arrious, à $2150 \mathrm{~m}$ d'altitude.

Nous avons le plaisir de dédier cet te espèce à $\mathbf{M}$. le Dr. P. Zwick, directeur de la station d'hydrobiologie de Schlitz en Hesse.

Clinocera zwicki est très proche de Cl. lyneborgi Vaillant et Chvála, mais en diffère par les caractères suivants : l'aile n'a ni tache stigmatique ni parties enfumées, alors qu'il y en a chez la seconde espèce; les lobes postérieurs des gonostyles sont beaucoup plus larges chez $\mathrm{Cl}$. lyneborgi que chez $\mathrm{Cl}$. zwicki; enfin les prolongements postérieurs des gonocoxites sont nettement plus courts chez $\mathrm{Cl}$. lyneborgi que chez $\mathrm{Cl}$. zwicki; il n'en reste pas moins que les deux espèces peuvent être réunies en un même groupe.
Les Clinocera du sous-genre Phaeobalia, dont font partie les deux espèces que nous venons de citer, sont orobiontes et fréquentent les parois rocheuses suintantes aux abords des glaciers ; on en observe parfois sur la glace de névés ou de glaciers (Vaillant 1973). Toutefois Clinocera (Phaeobalia) inermis (Loew), à vaste répartition altitudinale, peut seulement être considérée comme orophile (Vaillant 1964), ainsi que Cl.(Ph.) vaillanti Wagner, des Alpes Méridionales et des Apennins, dont la répartition altitudinale est moins étendue (Wagner 1982).

\section{Clinocera (Kowarzia) subplectrum n. sp.}

Imago $\sigma$ : Sur la face, 2 longues et 8 courtes soies. Corps brun, pattes claires. Les mêmes chètes sur le mesonotum et sur le scutellum que pour $C L$. $(K$. plectrum (Mik). Fémur I (fig. 5) : pas de chètes remarquables, à part 1 chète ventral et 1 chète antérieur, tous deux préapicaux, un peu plus longs que les autres ; peigne formé de 7 éléments, dont le demier est décalé par rapport aux autres. Aile (fig. 1): tache stigmatique extrêmement pâle; rapport stigmatique $: 6,6$; rapport cubital $: 4,90$; rapport postical : 1,06 ; angle cubital : $95^{\circ}$; angle postical : $53^{\circ}$; ner vure $\mathrm{p}_{2}$ anguleuse; deuxième segment de la ner. vure anale distinct, mais assez court ; un épaississement de la nervure cubitale à l'extrémité de son premier segment ; transverse $d_{2}$ et transverse dis. cale à peu près de même longueur ; la nervure $c_{2}$ aboutit à l'apex de l'aile. Genitalia (fig. 6 et 7 ) : cha. que gonostyle, en forme de faucille, se caractérise par la présence d'un talon antérieur peu important, à la différence de $C l$. (K.) plectrum (Vaillant 1964 : fig. $3 \mathrm{f}$ ), chez lequel ce talon est très développé. Le prolongement postérieur de chaque gonocoxite est ici rectiligne, comme pour $C l$. $(K$.) sandaliae Wagner, de Sardaigne, et comme pour $C l$. (K.) jalonae Wagner, du centre de l'Espagne ; l'hypandrium n'a qu'un seul chète près de son extrémité ; le premier segment de la partie libre de l'aedeagus est très épais, avec un bec distal caractéristique.

Longueur de l'aile : $3,6-3,7 \mathrm{~mm}$.

L'unique spécimen, l'holotype, a été capturé le 8 novembre 1984 en bordure d'un ruisseau à $500 \mathrm{~m}$ au nord de Béon, à $450 \mathrm{~m}$ d'altitude, au lieu dit "Miédougué ".

Cl. (K.) subplectrum fait partie d'un groupe d'espèces très proches, le groupe plectrum, qui comprend à not re connaissance 4 espèces ; nous les séparerons de la façon suivante: 
1 (2)-prolongement postérieur de chaque gonocoxite incurvé. . . . . . . . . Ct. (K.) plectrum (Mik) $\ldots \ldots \ldots \ldots \ldots$ Alpes, Carpates, Caucase

2 (1) - prolongement postérieur de chaque gonocoxite rectiligne . . . . . . . . . . . . . . . .

3 (4) - le prolongement postérieur de chaque gonocoxite a au moins $1 / 2$ longueur du gonostyle.......... $C l(K)$ jalonae Wagner. . Sierra de Guadarrama.

4 (3) - le prolongement postérieur de chaque gonocoxite a moins de la $1 / 2$ longueur du gonostyle......5

5 (6) - prolongement postérieur de chaque gonocoxite progressivement at ténué vers son extrémité, en vue de profil.....Cl. (K.) sandaliae Wagner Sardaigne

$6(5)$-prolongement postérieur de chaque gonocoxite d'épaisseur égale en vue de profil

Cl (K.) subplectrum n. sp. . . . . . . . . Pyrénées

Le genre Bergenstammia est établi en 1881 par Mik. E.O. Engel ne le considère que comme un sous-genre de Clinocera. Par la suite Bergenstammia est rétabli comme genre (Vaillant 1978, Wagner 1982 b). Ceci est tout à fait justifié du fait que les représentants des Bergenstammia partagent certains caractères avec les Clinocera, d'autres avec les Wiedemannia et ont également quelques traits particuliers :

Les Clinocera comme les Bergenstammia sont dépourvues de joues, du fait que leur péristome rejoint leurs yeux de chaque côté de la tête, alors que les Wiedemannia ont toujours des joues.

Les Wiedemannia et les Bergenstammia ont des chètes dc bien plus nombreux que les Clinocera; les représentants des deux premiers genres ont un pont tergal épais, qui s'élargit progressivement de chaque côté en rejoignant les gonocoxites (fig. 10 et 22), alors que les Clinocera ont un pont tergal grèle et d'épaisseur constante (fig. 13).

Chez les Bergenstammia, les tarses sont dépourvus de pulvilles et les prolongements postérieurs des gonocoxites sont décomposés chacun en une partie fixe et en une partie mobile, ce qui n'est le cas ni chez les Clinocera, ni chez les Wiedemannia. Les Bergenstammia sont de taille particulièrement grande parmi les Clinocerini.

\section{Bergenstammia pyrenaica $\mathrm{n}$. $\mathrm{sp}$.}

Des représentants de cette espèce ont été recueil. lis par P. Lavandier dans les Hautes-Pyrénées, de sorte que son nom spécifique figure dans le mémoire de cet hydrobiologiste, ainsi que dans un autre ouvrage (Vaillant 1978), mais il est demeuré jusqu'ici un nomen nudum. Imago $\sigma$ : Sur le mesonotum, pas d'acrosticales; de chaque côté, 10 ou $11 \mathrm{dc}, 1$ huméral, 1 présutural, 2 chètes dans le triangle sutural, 2 supraalaires à peu près de même longueur. Fémur I : sur le $1 / 3$ proximal, des soies claires ventrales, confusément bisériées et longues comme le
$1 / 2$ travers du fémur ; sur le $1 / 4$ distal (fig. 21). 3 chètes antérieurs alignés ; sur le $1 / 8$ distal, 2 ou 3 chètes postérieurs alignés obliquement. Aile : tache stigmatique pâle ; rapport stigmatique : 5,15 ; rapport cubital : 5,77 ; rapport postical : 0,82 ; angle cubital : $92^{\circ}$; angle postical $: 52^{\circ}$; premier segment de la nervure subcostale avec un court épaississement ; nervure $\mathrm{p}_{2}$ régulièrement incurvée; segment distal de la nervure anale aussi long que $d_{3}$; la nervure $d_{2}$ est très oblique par rapport à l'axe de l'aile et elle est un peu plus courte que la transverse discale; l'apex de l'aile est au $1 / 3$ de la distance entre l'extrémité de $c_{2}$ et celle de $d_{1}$. Genitalia (fig. 18, 19 et 22): gonostyles arrondis à leur extrémité et avec de longues soies sur leur face interne (fig. 23); partie mobile des prolongements postérieurs des gonocoxites avec deux lobes très inégaux (fig. 20), le postérieur étant peu développé.

Longueur de l'aile : 6,0-6,2 $\mathrm{mm}$.

L'holotype a été pris près du ruisseau d'Arrious à $2150 \mathrm{~m}$, le 22.9 .1985 et un autre exemplaire le même jour non loin de là, à $1775 \mathrm{~m}$.

Toutes les Bergenstammia connues jusqu'ici sont orobiontes et s'observent surtout sur la mousse détrempée et sur les rochers suintants dans les ruisseaux de haute montagne.

Voici une clé des quelques espèces européennes connues jusqu'ici :

1 (2) : pas de chètes particuliers sur la face antérieure de chaque fémur I

B. multiseta (Strobl) ............Alpes orientales

2 (1): plusieurs chètes alignés sur la face antérieure de chaque fémur $1, \ldots \ldots \ldots \ldots \ldots \ldots \ldots, \ldots, 3$

3 (4) : partie mobile du prolongement postérieur de chaque gonocoxite simple et globuleuse............. B. slovaca Wagner................. Tatras

4 (3) : partie mobile du prolongement postérieur de chaque gonocoxite bilobé ou grossièrement quadran. gulaire ................... 5

5 (6) : lobes de la partie mobile du prolongement postérieur de chaque gonocoxite presque de même longueur B. nudimana (Vaillant)...... Alpes occidentales.

$6(5)$ : lobes de la partie mobile du prolongement postérieur de chaque gonocoxite de longueur inégale ou tous deux très courts..................... 7

7 (8) :gonostyles coudés en vue latérale; partie mobile du prolongement antérieur de chaque gonocoxite quadrangulaire en vue latérale

B. frigida (Vaillant)........... Alpes occidentales

8 (7) : gonocoxites rectilignes en vue latérale; partie mobile du prolongemen postérieur de chaque gonocoxite

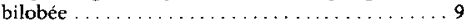

$9(10)$ : gonostyles acuminés à leur extrémité, vus de profil B. nudipes (Loew) Alpes orientales et occidentales 
10 (9) : gonostyles arrondis à leur extrémité . B. pyrenaica n. sp ............... Pyrénées

\section{Wiedemannia (Chamaedipsia) wachtli (Mik)}

Imago $\sigma$ : Joue haute comme le $1 / 4$ de la hauteur de l'œeil. Mésonotum : acrosticales petites, biseriées et s'étendant jusqu'à la dépression préscutellaire ; de chaque côté, $5 \mathrm{dc}$ et quelques chètules intercalaires, 1 huméral, 1 chète et 11 chètules présuturaux, 2 chètes et 6 chètules dans le triangle sutural, 2 supraalaires. Scutellum : 2 chètes et 6 chètules marginaux, 8 à 10 chètules sur le disque. Fémur I : 1 grand chète préapical antéricur. Aile : pas de tache stigmatique ; rapport cubital $: 7,00$; rapport postical : 1,57 ; angle cubital : $118^{\circ}$; angle postical : $43^{\circ}$; nervure $\mathrm{P}_{2}$ fortement incurvée ; deuxième segment de la nervure anale totalement absent; nervure $d_{2}$ de longueur variable et parfois deux nervures $d_{2}$ aux deux extrémités d'une petite cellule rectangulaire; l'apex de l'aile est à une distance de l'extrémité de $c_{2}$ égale à $1 / 4$ de la distance à l'extrémité de $d_{1}$. Genitalia : gonostyles chacun avec de nombreux rétinacles dans leur partie la plus proche du gonocoxite (fig. 11) ; prolongements postérieurs des gonocoxites de très petite taille, massifs en vue latérale ( $f i g .12)$, grèles de face ( $\mathrm{ig} .16$ ) et mobiles par rapport aux gonocoxites; cerques petits : sur le côté droit de la figure 10 , on voit 3 sclérites en dessous de chaque cerque et qui sont des apodèmes; hypandrium avec 2 ou 3 chètes seulement.

Longueur de l'aile : 3,1-3,2 mm.

W. wachtli est remarquablement commune dans le bassin du gave d'Ossau entre $495 \mathrm{~m}$ et $1775 \mathrm{~m}$ d'altitude et nous avons pu examiner plus de 100 spécimens $\sigma$ et $q$; l'espèce serait plus rare dans les Hautes-Pyrénées.

Le type de $W$. wachtli provient des Alpes orientales et aucun spécimen n'a été capturé jusqu'ici dans les Alpes occidentales et dans le Massif Central. Cette discontinuité dans l'aire de répartition de cette espèce paraît suspecte et on peut se demander si les W. wachtli des Pyrénées n'appartiennent pas en réalité à une espèce distincte de celle des Alpes orientales, bien que les genitalia $\sigma$ apparaissent semblables. En effet, si l'on se reporte à la description de E.O. Engel, on s'aperçoit que la véritable $\boldsymbol{W}$. wachthi n'a pas de chète préapical antérieur sur le fémur I (ce qui l'a fait placer dans le sous-genre Philolutra) et a une tache stigmatique faiblement pigmentée sur l'aile. Malheureusement nous n'avons pu jusqu'ici nous procurer de spécimens des Alpes et nous admettrons provisoirement que $W$. (Ch.) languedocica Vaillant est synonyme de W. (Ch.) wachtli (Mik).
Wledemannia (Chamaedipsia) berthelemyi $n$. sp.

Imago $\sigma^{*}$ : Joue haute comme les $2 / 3$ de la hauteur de l'œil, qui semble particulièrement petit. Mesono tum : 8 à 10 acrosticaux bisériés ; de chaque côté 5 dc sans intercalaires, 1 huméral, 5 chètes présuturaux, dont 2 sont bien plus développés que les autres, 2 chètes et 5 minuscules chètules dans le triangle sutural, 2 supraalaires. Scutellum : 2 chètes et 4 chètules marginaux, au moins 15 chètules sur le disque. Fémur I : sur le $1 / 3$ proximal, une ciliation ventrale, dont les éléments sont longs comme le $1 / 2$ travers du fémur: 1 à 3 chètes préapicaux antérieurs, dont 1 au moins est de grande taille, et 1 préapical ventral (fig. 24). Aile : pas de tache stigmatique ; rapport cubital : 8,39 ; rapport postical : 1,46 ; angle cubital : $120^{\circ}$; angle postical : $40^{\circ}$; nervure $\mathrm{p}_{2}$ fortement incurvée; deuxième segment de la nervure anale totalement absent ; transverse discale et nervure $p_{1}$ de même longueur: il n'y a pas de nervure $d_{2}$, mais par contre un segment de nervure $d+d_{3}$ long, chez les 2 exemplaires examinés, comme les $2 / 3 \mathrm{de}_{3}$. Genitalia: les gonostyles ont chacun une forte encoche sur leur bord libre et, sur leur face interne, des chètes courbes, incurvés et irrégulièrement répartis ( $\mathrm{fig}$. 25) ; le prolongement postérieur de chaque gonocoxite est très petit et nous n'avons pas réussi à le mettre en évidence ; hypandrium sans chètes (fig. 32).

Longueur de l'aile : $3,9 \cdot 4,0 \mathrm{~mm}$.

L'holotype provient d'un ruisseau à $500 \mathrm{~m}$ au nord de Bèon, à $450 \mathrm{~m}$ d'altitude, au lieu-dit "Miédougué ", le 28-9-1984.

Nous avons dédié cette espèce à M. Cl. Berthélemy, professeur à l'université de Toulouse.

W. berthelemyi se place à part parmi les espèces du sous-genre Chamaedipsia par la forme très particulière de ses gonostyles.

\section{Wedemannia (Chamaedipsia) digitata n. sp.}

Imago $\sigma$ : Face saillant en museau, avec une dépression médiane et un bourrelet la limitant sur les côtés et en haut ; joue haute comme les $2 / 7$ de la hauteur de l'œil. Mésonotum: plus de 20 acrosticaux de grande taille; de chaque côté, 5 dc avec seulement 1 ou 2 chètules intercalaires, 1 huméral, 5 présuturaux, dont les 2 antérieurs sont très rapprochés l'un de l'autre et dont les $\mathbf{2}$ inférieurs sont plus petits que les autres, 2 chètes et 5 chètules dans le triangle sutural, 2 supraalaires. Scutellum : 2 chètes et 4 chètules 


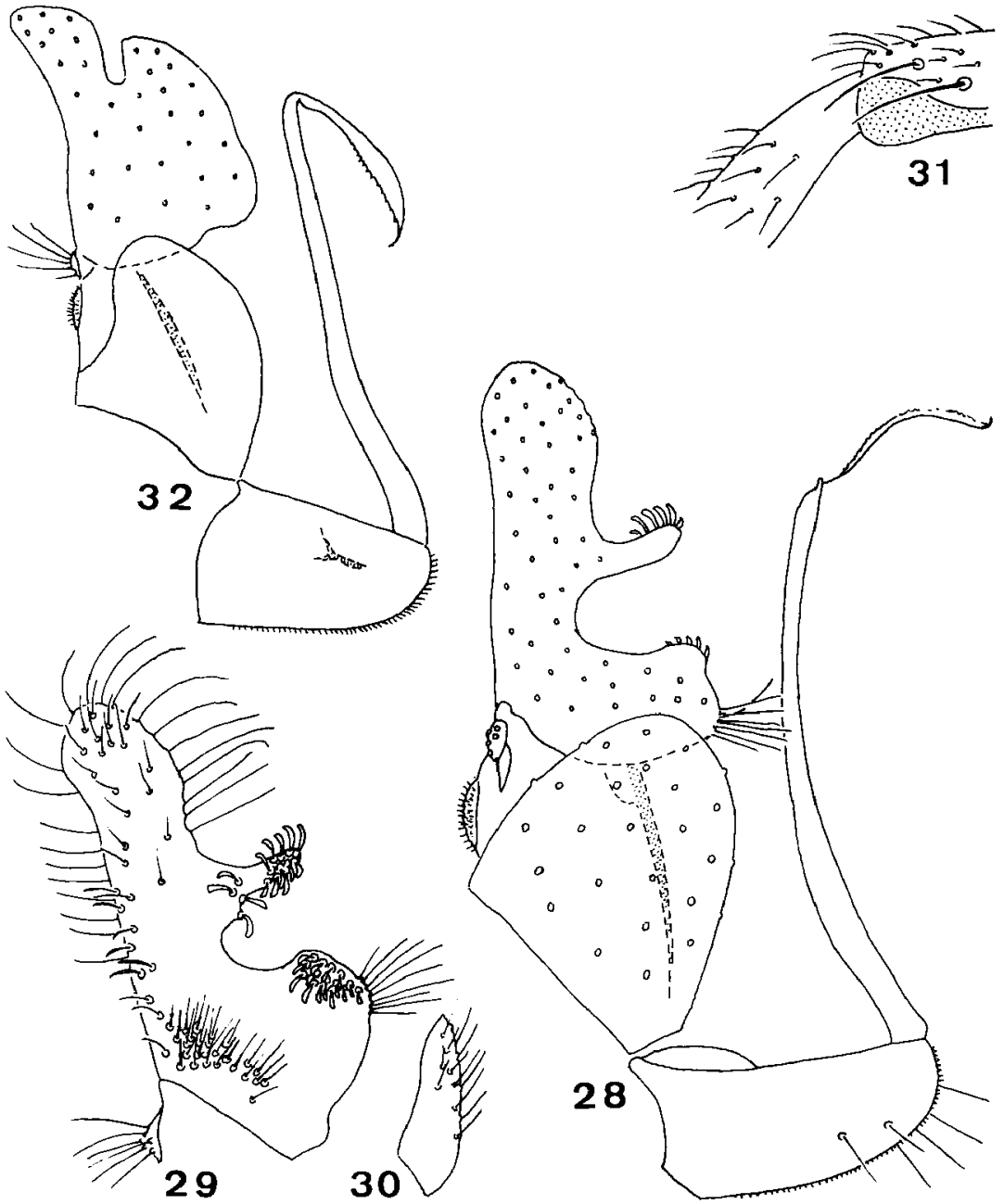

Fig. 28 - 32 : imagos mâles - 28 - 31 : Wiedemannia digitata n. sp. - 28 : genitalia, profil - 29 : gonostyle droit, face interne - 30 : prolongement postérieur du gonocoxite droit, face interne - 31 : partie distale du fémur I droit, face antérieure $\cdot 32$ : Wiedemannia berthelemyi $\mathrm{n}$. sp., genitalia, profil. 
marginaux ; plus de 20 chètules sur le disque. Fémur 1 : une ciliation ventrale dense, dont les éléments ont environ $1 / 3$ du travers du férmur ; sur la $1 / 2$ ou le $1 / 3$ distal, 2 ou 3 chètes antérieurs alignés, dont l'un est généralement préapical. Aile : cellule anale très particulière du fait que la nervure $p_{2}$ est faiblement incurvée et fortement récurrente; deuxième segment de la nervure anale bien marqué et un peu plus long que $\mathrm{p}_{2}$; transverse discale un peu plus courte que $\mathrm{p}_{1}$; comme pour $W$. berthelemyi, il n'y a pas de transverse $d_{2}$, mais un segment de nervure longitudinale $d+d_{3}$, dont la longueur, assez variable, est généralement voisine de celle de $\mathrm{p}_{2}$; l'apex de l'aile est à une distance de l'extrémité de $c_{2}$ comprise entre le $1 / 3$ et le $1 / 4$ de sa distance à l'extrémité de $d_{1}$. Genitalia : gonostyles (fig. 29) avec chacun 2 lobes s'étendant vers l'arrière, recourbés et portant l'un et l'autre sur leur face interne des rétinacles groupés ; ces gonostyles ont également un groupe de soies internes et un autre groupe postérieur ( $f$ ig. 27) près de leur base; le prolongement postérieur de chaque gonocoxite, articulé sur celui-ci, est grèle vu de face (fig. 27) comme de profil (fig. 30) : hypandrium assez long, avec 10 à 12 chètes dispersés (fig. 28).

Longueur de l'aile : 4,2 - 4,3 mm.

Neuf spécimens o ont été examinés. L'holotype a été pris le 19.9.1983 en bordure du ruisseau d'Arrious à $2150 \mathrm{~m}$.

\section{Discussion}

Si nous ne tenons pas compte des nomina nuda et des espèces trouvées dans les Pyrénées, mais non encore signalées d'une façon précise, le nombre des espèces de Clinocerini représentées dans ce grand ensemble montagneux est de 30 , ce qui est peu en comparaison avec les Alpes; mais bien d'autres espèces seront certainement découvertes dans les Pyrénées au cours des années à venir, si des entomologistes veulent bien s'intéresser à ces insectes.

Sur les 30 espèces, 15 ont une vaste répartition en Europe et sont représentées pour le moins à la fois dans les Alpes, les Carpates et les Pyrénées ; ce sont Dolichocephala guttata (Haliday), D. irrorata (Fallèn), Clinocera appendiculata (Zetterstedt), $\mathrm{Cl}$. schremmeri (Vaillant) $\left(^{*}\right), \mathrm{Cl}$. barbatula (Mik), $\mathrm{Cl}$. bipunctata (Haliday), Cl. stagnalis (Hal.) $\left({ }^{\star}\right), \mathrm{Cl}$. wesmaeli (Macquart), Wiedemannia beckeri Mik, W. bistigma (Curtis), W. bohemani (Zetterstedt), W. fallaciosa (Loew), W. hygrobia (Loew), W. ouedorum
Vaillant et $W$. oxystoma Bezzi. L'avant-dernière de ces espèces se retrouve en Afrique du Nord et $W$. fallaciosa a un territoire très étendu qui s'étend jusqu'en Asie centrale et en Afrique septentrionale. Une seule des 15 espèces, Wiedemannia beckeri, est franchement orobionte; les 14 autres sont oroxènes ou faiblement orophiles, bien qu'elles aient, pour la plupart, une vaste répartition altitudinale.

Seulement 5 espèces, sur les 30, Dolichocephala austriaca Vaillant, Clinocera madicola (Vaillant), Bergenstammia nudipes (Loew), Wiedemannia aerea Vaillant et $W$. wachti (Mik) ne sont représentées que dans les Alpes et les Pyrénées et une seule, Wiedemannia mirousei Vaillant, ne se rencontre que dans le Massif Central et les Pyrénées. Bergenstammia nudipes et Wiedemannia aerea sont orobiontes et les 4 autres espèces fortement orophiles.

Les endémiques pyrénéennes de Clinocerini sont pour le moins au nombre de 9 ; ce sont Clinocera subplectrum n. sp., Cl. zwicki n. sp., Bergenstammia pyrenaica n. sp., Wiedemannia angelieri Vaillant, $W$. berthelemyi n.sp., W. digitata n.sp., W. falcifera Vaillant, $W$. oredonensis Vaillant et $W$. thomasi Vaillant. Toutes sont orobiontes ou fortement orophiles et leur endémisme est sans doute dû à leur isolement orogène ; pourtant Clinocera subplectrum et Wiedemannia berthelemyi, dont les types ont été trouvés dans un ruisseau de basse altitude, très froid (température maximale : $9^{\circ} \mathrm{C}$ le 27.7 .1984 ), font exception. Parmi les endémiques pyrénéennes, certaines ont assurément des affinités avec des espèces de la péninsule ibérique ; c'est manifestement le cas pour Clinocera zwicki; nous n'avons malheureusement encore que très peu de documents concernant les Empididae d'Espagne et du Portugal.

Cette étude nous a permis de comparer les ailes de plusieurs espèces de Clinocerini à l'aide d' " indices "; nous avons pu constater que, malgré une uniformité apparente de leur nervation, les ailes des Clinocerini sont fort diversifiées et que leurs caractères peuvent être utilisés en taxonomie d'une façon très efficace.

Nous avons pu également comparer les genitalia mâles de 6 espèces de Clinocerini appartenant à 3 genres différents. Nous avons utilisé la nomenclature de $\mathrm{H}$. Ulrich basée sur une étude de la musculature de la partie postérieure du corps chez une

(*). G. Vinçon (1987) 
espèce de la tribu. Son interprétation des pièces a tout lieu de surprendre, car elle est très différente de celle qu'il fournit pour les pièces génitales des Empididae de la tribu des Hemerodromini (Ulrich 1975) ; ces derniers auraient deux grands cerques en forme de lames, garnis de rétinacles et rappelant les gonostyles des Clinocerini. Un argument pourrait être fourni en faveur de l'interpretation d'UIrich : si l'on examine les figures des genitalia mâles exécutées par E.O. Engel pour Clinocera (Phaeoba(ia) dimidiata Loew et pour $C l$. (Ph.) pokornyi (Mik), on peut voir, en avant des gonostyles sensu Ulrich, deux pièces allongées et ciliées, qui représentent sans doute deux cerques, non plus en forme de petites plaques, mais sous l'aspect de véritables appendices.

\section{Travaux cités}

Bährmann (R.) 1960. Vergleichend-morphologische Untersuchungen der männlichen Kopulationsorgane bei Empididen (Diptera). Beitr. Ent. Berlin, $10: 485.540$.

Collin (J.-E.) 1961. British flies, VI, Empididac. VIII +782 pp. University Press, Cambridge.

Engel (E.-0.) 1938-1952. Empididae. in Lindner E. : Die Fliegen der palaearkischen Region, Bd IV $+: 1$ - 400, Taf. 1-34. Schureizer bart, Sultgart.
Lavandier (P.) 1979. Ecologie d'un torrent pyrénéen de haute montagne. l'Estaragne. Thèse de Doctorat d'Etat, Toulouse III : $532 \mathrm{p}$ -

Ulrich (H.) 1972. Zur Anatomie der Empididen-Hypopygiums (Diptera). Veröff. zool. Staatssamml. München, 16:1-27.

Ulrich (H.) 1975. Das Hypopygium von Chelifera precabunda Collin (Diptera, Empididae). Bonn. zool. Beitr., 26: 264.279.

Vaillant (F.) 1964. Revision des Empididac Hemerodromiinae de France, d'Espagne et d'Afrique du Nord (Dipt.). Ann. Soc. ent. Fr. 133:143.171

Vaillant (F.) 1967. La repartition des Wiedenamnia dans les cours d'eau et leur utilisation comme indicateurs de zones écologiques (Diptera, Empididae). Annls Limmol, 3 (2) : 267-293.

Vaillant (F.) 1968. Quelques Empididae Hemerodromiinae des Pyrénées (Diptera). Annls Limnol, 4 (1) : 85-93.

Vaillant (F.) 1973. Oueiques insectes Dipteres, à larves aquatiques, du parc de la Vanoise. Trav. scient. Parc National Vanoise, 3: 133-165.

Vaillant (F.) \& Chvàla (M.) 1973. Empididae Hemerodromiinae from Spain (Insecta, Diptera). Steensinupia, Coperthagen, 3 (6) : 57-64

Vaillant (F.) 1978. Empididae. in Illies (J.) : Limnofauna Europaea 2 Aufl. : $465-469$. Fischer. Stuugart

Vinçon (G.) 1987. Comparaison de la taune benthique des vallées d'Aure et d'Ossau, en vue de l'élaboration d'une méthodologie de surveillance des cours d'eau de montagne. These de Docteur Ingénieur, Université Toulouse III : 381 p.

Wagner (R.) 1982a. Ueber einige Empididae (Clinoceratinae) aus den Alpen und aus italien (Diptera, Empididae). Zeitschr. $f$ Entom. Linz, 3 (15) 217-224.

Wagner (R.) 1982b. Neure und stilsent paläarktische Tanzfliegen (Diptera, Empididae). Acia ew buhlencslow, $81: 458-464$.

Wagner (R.) 1984. Two new species of Mediterranean Empididae (Diptera). Boll. Soc. ent, iial., Genova, $116(4-7): 121-123$. 